\title{
Google Trends Topic-Based Uncertainty: A Multi-National Approach
}

\section{Florian Schütze}

Department of Socioeconomics, University of Hamburg, Germany.

\begin{abstract}
Several studies have shown that uncertainty among economic actors influences business cycle dynamics. This paper uses Google Trends topic queries to construct an uncertainty proxy that can be applied to every country where Google is active. Using a VAR approach, this paper demonstrates that the obtained impulse-response functions of main economic indicators to a onestandard deviation shock to the constructed indicator, are similar to those from an already-existing uncertainty proxy, the EPU. This is true for the G7 countries and Russia. On average, the uncertainty indicator constructed for this paper leads to more statistically significant responses than does the EPU. Thus, this paper shows that Google Trends is a helpful tool for obtaining timely information about uncertainty among economic actors. The main improvement in this uncertainty proxy is in its language independence. Existing uncertaintymeasurement approaches, in contrast, rely on certain keywords that often vary across countries.
\end{abstract}

Keywords: Google Trends; Uncertainty; Business Cycle Dynamics; VAR. 


\section{Introduction}

A variety of authors have demonstrated that uncertainty among economic actors has an influence on business cycle dynamics (Federal Open Market Committee, 2009; Gilchrist et al., 2014; Fernández-Villaverde et al., 2015; Jones \& Olson, 2015; Segal et al., 2015; Leduc \& Liu, 2016; Shoag \& Veuger, 2016; Moore, 2017; Mumtaz \& Theodoridis, 2018). Uncertainty is a latent variable and cannot be directly observed, so there are three indirect measurement methods: measuring volatility in various metrics, such as forecast errors (Jurado et al., 2015) and stocks (Bloom, 2009); measuring dispersion among forecasters or in business surveys about economic tendencies (Bachmann et al., 2013); and measuring the occurrence of keywords in certain mediums, such as newspapers, as it is done by Baker et al. (2016) for the Economic Policy Uncertainty index (EPU).

In this paper, an uncertainty indicator based on the third method is constructed using Google Trends search topics. This indicator can be applied directly to all countries that use Google. The advantages of this approach over the EPU are threefold. First, it does not rely on journalists writing about their own perceptions of economic uncertainty-it measures it directly from economic actors. Second, it is not only limited to uncertainty over economic policy - it covers overall economic uncertainty. Third, it can be conducted daily in real time.

The results of this paper show that shocks to the constructed uncertainty proxy in the G7 countries and Russia lead to similar VAR impulse-response functions compared to shocks to the EPU. On average, the uncertainty indicator constructed for this paper leads to more statistically significant responses than does the EPU. The difference in significant responses between them is also, in turn, statistically significant.

Previously, the use of Google Trends to construct an uncertainty indicator has been done primarily with keywords (e.g., Castelnuovo \& Tran, 2017; Bontempi et al., 2018; Donadelli $\&$ Gerotto, 2019). The works that have employed this method all show that their proxy influences business cycle dynamics while providing information quickly, relative to other uncertainty proxies. The problem with this approach, however, remains-for every different language, there must be a unique uncertainty-related word set.

Fortunately, Google Trends offers the ability to search for topics rather than keywords. Thus, Google presents a serious advantage; it uses machine-learning techniques to identify the underlying topics in search queries. With this option, extending the uncertainty proxy to every Google-using country is fairly straightforward, provided that the relevant topics are identified. Until now, this approach had only been used by Kupfer and Zorn (2019) with ten topics and four categories; no selection criteria for the topics or categories were provided. Nonetheless, they found a statistically significant influence on stock market volatility. 
This paper is structured as follows. The following section provides insight into the theoretical background on uncertainty's importance matters; it also explains why the Google Trends approach is suited for this task. The third section offers an overview of the empirical procedure used for this paper. The fourth section presents the results of the VAR analysis. Finally, the fifth section concludes the paper.

\section{Theoretical Background}

The idea that Google Trends can accurately measure economic uncertainty relies on two assumptions. First, uncertain actors aim to reduce their uncertainty by gathering information on a certain subject. Second, actors using the Internet to gather information do so primarily using search engines, among which Google is the most significant. With these two assumptions, one can conclude that higher search requests reflect higher uncertainty.

In terms of the theoretical background, the reason uncertainty has a negative influence on the economy is twofold (Bloom, 2014). On the one hand, consumers and investors may postpone actions when uncertainty is high. This is called the "wait-and-see" effect when said postponement is accompanied by an overshooting of consumption and investment after uncertainty levels return to normal. On the other hand, uncertainty may be interpreted as constituting a high risk ${ }^{1}$ and, therefore, overall risk premiums rise, leading to an increase in the cost of investments.

A VAR is used to estimate impulse-response functions of bonds, shares, and industrial production after a shock of uncertainty. The response of bond yields to a shock of uncertainty can be positive or negative, depending on the attitude of economic actors toward the government. A positive response suggests that people demand a risk premium due to high uncertainty. A negative response suggests that people have high trust in their governments and buy these bonds, leading to a decrease in the yield. It is assumed that elevated uncertainty negatively impacts stock market returns because people sell their shares with a "wait-andsee" attitude. It is also assumed that high uncertainty negatively impacts industrial production.

\section{Empirical Approach}

\subsection{Data}

This paper used data from Google Trends for its empirical analysis. The data was obtained using the "gtrendsR" package for the program "R." Data on stock market returns, long-term

\footnotetext{
${ }^{1}$ Here, it is important to distinguish uncertainty from risk. See Knight (1921) for definitions of risk and uncertainty.
} 
government bond yields, and industrial production for the G7 countries and Russia is also used (Data source: OECD, 2019c; OECD,2019b; OECD, 2019a). All data was integrated of order one to obtain stationary time-series and seasonal-adjusted data using "X-13ARIMASEATS."

For stock market returns, this paper uses the major stock index of each country measured in monthly growth rates. For changes to long-term government bond yields, bonds with a maturity of ten years are used. Industrial production is represented by annualized monthly growth rates of said industrial production.

The period considered for the G7 countries is from 02/2004 to 02/2019; for Russia, the period is from 02/2004 until 06/2018, as the OECD only has long-term Russian government bond data up to $06 / 2018$.

\subsection{Construction Approach}

To identify economic uncertainty-related topics, this paper used 184 Google Trends queries stemming from Bontempi et al. (2018), which are based on category-specific policy keywords by Baker et al. (2016). The underlying topics were identified by inserting the keywords into Google Trends and saving the first returned topic. After deleting recurring ones, the process ultimately returned 156 topics. Additionally, the four news categories suggested by Kupfer and Zorn (2019) were also included.

These 160 potential search queries were then downloaded for all countries being considered. Nineteen queries that returned no data for at least one considered country were excluded, leaving us with 141 queries.

In order to keep only queries that are highly informative, the data for the US and Canada is used as a training set. As the initial keywords from Baker et al. (2016) were crafted for the construction of the EPU from US newspapers, the topic selection needed to be done with US data. However, since this could lead to very US-specific topics, Canadian data was included to mitigate this effect.

Ultimately, 141 US and Canadian VARs were estimated in the following order: a single query out of all 141; long-term government bond yields; stock market returns; industrial production. Since we are interested in the contemporaneous effect of the resulting impulse-response functions, the transmission is assumed to follow this order. The lag length of the VAR was chosen by AIC with a maximum lag of 12 months.

For construction, only topics and categories with at least three statistically significant responses of the US and Canadian VAR are kept. The corresponding impulse-response functions stem from how stocks, bonds and industrial production respond to a one-standard deviation shock in a Google Trends query. 
The in the paragraph above mentioned procedure resulted in 13 queries: twelve topics and one news category. Only one query, namely the news category, is identical to the 14 queries used by Kupfer and Zorn (2019). To construct the Google Trends Topic Uncertainty indicator (GTTU) for each country, the remaining queries were aggregated in accordance with the suggestion made by Bontempi et al. (2018) to obtain uncertainty indicators for each country.

A VAR was constructed for the G7 countries and Russia in the following order: the countryspecific aggregated GTTU; country-specific long-term government bonds; country-specific stock market returns; country-specific industrial production. Once again, the lag length of the VAR was chosen by AIC with a maximum lag of 12 months.

\section{Country-Specific Results}

Figure 1 is a comparison between this paper's US GTTU (GTTU_US) and the US EPU (EPU_US) from Baker et al. (2016). The GTTU shows a sharp spike in uncertainty around the 2008/2009 financial crisis and two smaller spikes in 2016 and 2018. The EPU shows spikes around the 2008/2009 financial crisis as well as in 2012 and 2016, which could reflect political uncertainty regarding US elections. It also shows a clear spike in 2018 , which is likely attributable to the US-China "trade war."
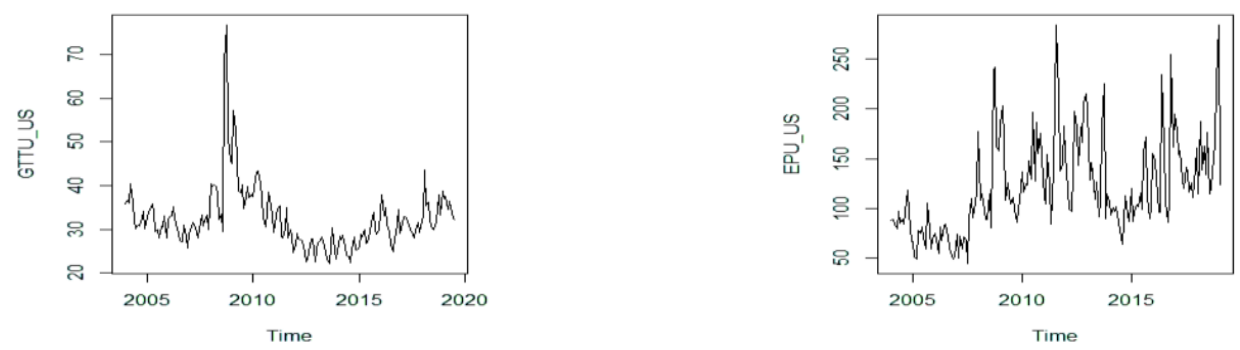

Figure 1. Uncertainty over time in the USA (GTTU_US vs. EPU_US).

Figure 2 presents the impulse responses to a one-standard deviation shock to uncertainty for all of the considered countries. Once more, it displays this paper's GTTU and the EPU. 
(a) United States

Orthogonal Impulse Response from GTU_US

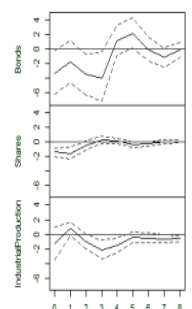

(c) France

Orthogonal Impulse Response from GTTU_FR

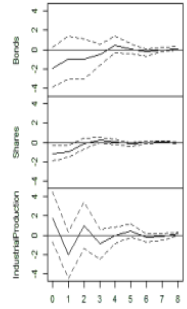

$95 \%$ Bootstrap Cl, 5000 runs

(e) Italy

Orthogonal Impulse Response from GTU _T

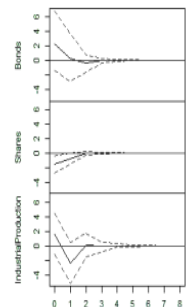

$95 \%$ Bootstrap Cl, 5000 runs

(g) United Kingdom

Orthogonal Impulse Response from GTIU_UK

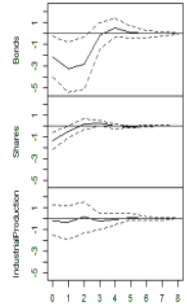

$95 \%$ Bootstrap Cl, 5000 runs
Orthogonal Impulse Response from EPU_US

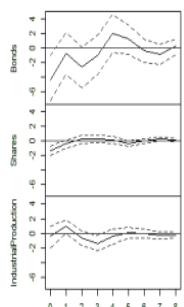

Orthogonal Impuse Response from EPU_FF

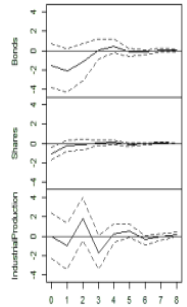

$95 \%$ Bootstrap Cl, 5000 runs

thogonal Impulse Response from EPU IT

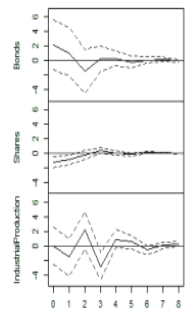

$95 \%$ Bootstrap Cl, 5000 runs

Othogonal Impuse Response from EPU_UK

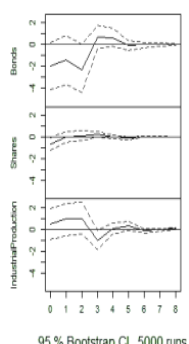

(b) Canada

Orthogonal Impulse Response from GTIU_CA

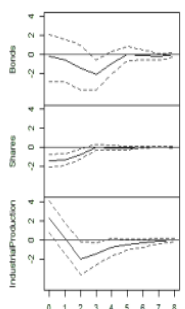

(d) Germany enan $_{\text {- }}$

Orthogonal Impulse Response from GTTU DE

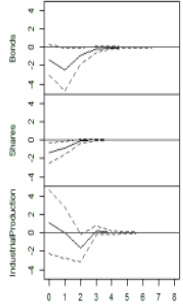

$95 \%$ Bootstrap Cl, 5000 runs

(f) Japan

Orthogonal Impulse Response from GTTU_JP

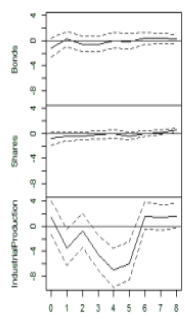

$95 \%$ Bootstrap Cl, 5000 runs

(h) Russia

Orthogonal Impulse Response from GTTU_RU

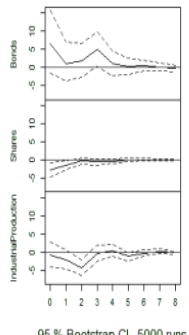

Orthogonal Impulse Response from EPU_RU

Othogonal Impulse Response from EPU_CA

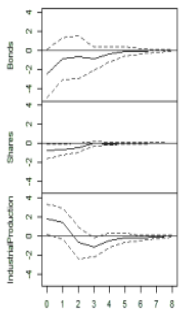

Orthogonal Impulse Response from EPU DE

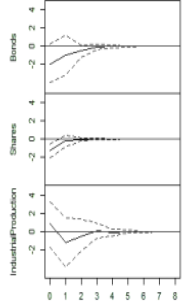

$95 \%$ Bootstrap Cl. 5000 runs

Othogonal Impulse Response from EPU JPP

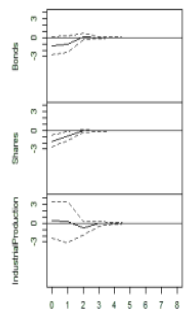

$95 \%$ Bootstrap Cl, 5000 runs

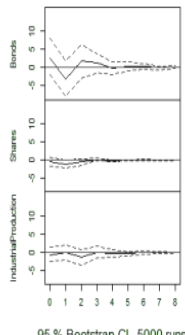

Figure 2. Impulse-response functions to a one-standard deviation shock to uncertainty. 
For the US, the responses are similar for both uncertainty indicators, though the GTTU offers more statistically significant responses (ssr) than does the EPU (a total of 13 GTTU ssr compared to 4 EPU ssr). The results for Canada are very similar, the only difference being that shares and industrial production show a stronger significant response from a GTTU shock compared to an EPU shock (7 GTTU srr, 4 EPU ssr). The two uncertainty measurements show similar results for France (3 GTTU ssr, 1 EPU ssr). German results indicate that, for bonds, only the GTTU shows a significant response. For shares, the significant response is one month longer for the GTTU. Industrial production shows a significant response in the second month for the GTT (5 GTT ssr, 1 EPU ssr).

For Italy, the statistically significant responses for shares are one month longer for the EPU. The other two responses show the same directions for both proxies, though they are only for the EPU and industrial production significant(2 GTTU ssr, 5 EPU ssr). The Japanese case is an outlier, as it shows a very strong response from industrial production to a GTTU shock (5 GTTU ssr, 2 EPU ssr). For the UK, the main difference in the impulse response functions lies in the response of bonds to a GTTU shock, which is significant for up to two months (5 GTTU ssr, 3 EPU ssr). For Russia, there is a significant response from bonds, shares, and industrial production to a GTTU shock; in contrast, an EPU shock results only in a significant response from shares (4 GTTU ssr, 1 EPU ssr).

In conclusion, similar directions of impulse responses to shocks to the EPU and the GTT suggest that they are both measuring the same hidden uncertainty. While stocks and bonds react alongside uncertainty shocks, industrial production generally reacts after about two months. For all countries considered, the response's direction is in line with the theoretical prediction. Interestingly, the reaction of government bonds is negative in all but Italy and Russia, suggesting that economic actors demand a risk premium amid high uncertainty in both countries. Importantly, the GTTU outperformed the EPU in terms of statistically significant responses in all of the considered countries except Italy. A one-standard deviation shock of the GTTU uncertainty proxy results in, on average, 5.5 statistically significant responses. The same shock to the EPU results in an average of just 2.63 statistically significant responses. Therefore, the GTTU garners, on average, 109\% more statistically significant responses than does the EPU, and this difference is significant at the $5 \%$ level. The difference for each impulse-response category is significant at least at the $10 \%$ level. Furthermore, the GTTU shows a significant response of share returns to uncertainty shocks for all considered countries. For five countries, it shows a significant response of bond yields and industrial production. In contrast, the Google Trends topic-based uncertainty proxy constructed by Kupfer and Zorn (2019) yielded far fewer significant responses (shares: US, UK, and France; bonds: US and UK; industrial production: Canada). 


\section{Conclusion}

This paper detailed the construction of an uncertainty indicator based on Google Trends topics. As shown, the resultant GTTU uncertainty proxy resembles the EPU but performs better - in terms of significant impulse responses - in seven of the eight countries considered. While Kupfer and Zorn (2019) had already produced a Google Trends topic-based uncertainty-measurement technique, they presented no procedure for choosing the topics. In this paper, the selection was based on the category-specific policy terms set by Baker et al. (2016). This resulted in 141 unique topics, which were further reduced by using the US and Canadian economic data collectively as a learning set in order to only keep topics relevant to the business cycle. Ultimately, 13 highly informative topics came to make up the Google Trends Topic Uncertainty indicator for each country.

With this uncertainty measurement, it is possible to construct a timely indicator for every country where Google is used, regardless of language. For example, when using English keywords for Japanese Google requests, only English-writing users would be considered. Furthermore, ambiguous keywords that may be present in some languages but not in others are not a concern, as all relevant keywords should be included in the topics.

The GTTU crafted for this paper exhibits behavior similar to that of the EPU from Baker et al. (2016). Moreover, it produces more statistically significant impulse responses than does the EPU, and this difference is statistically significant.

With the options provided by Google Trends, this indicator can operate on a daily basis. In addition to countries, it could apply to subregions like US states. There is a multitude of potential applications for this indicator that future research should consider. For example, researchers could use it to examine how citizens view their governments. As shown in the last chapter, reaction directions from government bond yields vary by country; these differences could be interpreted as differences in public attitude toward the state.

\section{References}

Bachmann, R., Elstner, S., \& Sims, E. R. (2013). Uncertainty and Economic Activity: Evidence from Business Survey Data. American Economic Journal: Macroeconomics, 5(2), 217-249.

Baker, S. R., Bloom, N., \& Davis, S. J. (2016). Measuring economic policy uncertainty. The Quarterly Journal of Economics, 131(4), 1593-1636.

Bloom, N. (2009). The Impact of Uncertainty Shocks. Econometrica, 77(3), 623-685.

Bloom, N. (2014). Fluctuations in Uncertainty. Journal of Economic Perspectives, 28(2), $153-176$. 
Bontempi, M. E., Frigeri, M., Golinelli, R., \& Squadrani, M. (2018). Uncertainty, Perception and Internet, Working Papers wp1134, Dipartimento Scienze Economiche, Universita' di Bologna.

Castelnuovo, E. \& Tran, T. D. (2017). Google It Up! A Google Trends-based Uncertainty index for the United States and Australia. Economics Letters, 161, 149-153.

Donadelli, M. \& Gerotto, L. (2019). Non-macro-based Google searches, uncertainty, and real economic activity. Research in International Business and Finance, 48, 111-142.

Federal Open Market Committee (2009). Minutes of the Federal Open Market Committee: December 15-16, 2009.

Fernández-Villaverde, J., Guerrón-Quintana, P., Kuester, K., \& Rubio-Ramírez, J. (2015). Fiscal Volatility Shocks and Economic Activity. American Economic Review, 105(11), 3352-3384.

Gilchrist, S., Sim, J. W., \& Zakrajsek, E. (2014). Uncertainty, Financial Frictions, and Investment Dynamics: Working Paper.

Jones, P. M. \& Olson, E. (2015). The International Effects of US Uncertainty. International Journal of Finance \& Economics, 20(3), 242-252.

Jurado, K., Ludvigson, S. C., \& Ng, S. (2015). Measuring uncertainty. American Economic Review, 105(3), 1177-1216.

Knight, F. H. (1921). Risk, uncertainty and profit. Boston: Houghton Mifflin.

Kupfer, A. \& Zorn, J. (2019). A Language-Independent Measurement of Economic Policy Uncertainty in Eastern European Countries. Emerging Markets Finance and Trade, 4(1), $1-15$.

Leduc, S. \& Liu, Z. (2016). Uncertainty shocks are aggregate demand shocks. Journal of Monetary Economics, 82, 20-35.

Moore, A. (2017). Measuring Economic Uncertainty and Its Effects. Economic Record, 93(303), 550-575.

Mumtaz, H. \& Theodoridis, K. (2018). The Changing Transmission of Uncertainty Shocks in the U.S. Journal of Business \& Economic Statistics, 36(2), 239-252.

OECD (2019a). Industrial production (indicator). Main Economic Indicators (database): DOI: 10.1787/data00052-en (Accessed on 18 July 2019).

OECD (2019b). Long-Term Government Bond Yields (10-years). Main Economic Indicators (database): DOI: 10.1787/data-00052-en (Accessed on 18 July 2019).

OECD (2019c). Share Prices (Indicator). DOI: 10.1787/6ad82f42-en (Accessed on 18 July 2019).

Segal, G., Shaliastovich, I., \& Yaron, A. (2015). Good and bad uncertainty: Macroeconomic and financial market implications. Journal of Financial Economics, 117(2), 369-397.

Shoag, D. \& Veuger, S. (2016). Uncertainty and the geography of the great recession. Journal of Monetary Economics, 84, 84-93. 